\title{
A FIT Formulation of Bianisotropic Materials over Polyhedral Grids
}

\author{
Piergiorgio Alotto ${ }^{1}$, Lorenzo Codecasa ${ }^{2}$ \\ ${ }^{1}$ Dip. Ing. Industriale, Università di Padova, Via Gradenigo 6/a, 35131, Padova, Italy, piergiorgio.alotto@ unipd.it \\ ${ }^{2}$ Dip. di Elettronica e Informazione, Politecnico di Milano, Via Ponzio 34/5, 20133 Milano, Italy, codecasa@elet.polimi.it
}

\begin{abstract}
Modeling bianisotropic constitutive equations, i.e. magnetoelectric coupling, in electromagnetics simulation increasingly important, in particular in metamaterials applications. This paper introduces for the first time such constitutive relationships in the framework of FIT and, furthermore, does so by allowing full generality in the discretization through arbitrary polyhedral grids. The resulting formulation is consistent, stable and preserves the thermodynamic properties of the bianisotropic constitutive equations thanks to the energetic approach used to construct the interpolating functions.
\end{abstract}

Index Terms-Must be chosen from the list of the IEEE keywords.

\section{INTRODUCTION}

The study of bianisotropy, i.e. magnetoelectric coupling in the constitutive equations, has been gaining increasing attention in recent years due to the possibility of practically obtaining it through metamaterials [1]. Although the mathematical properties of such constitutive equations have been known for decades [2], and the issue of the approximability of Maxwell's equations in the presence of bianisotropic materials has been addressed in the Finite Element context [3], no such investigations have been attempted in the framework of the Finite Integration Technique (FIT). The purpose of this work is to show how bianisotropic constitutive equations can be represented in FIT formulations over polyhedral grids by extending the energetic approach introduced in [4].

\section{DiscRETIZATION OF BIANISOTROPIC CONSTITUTIVE EQUATIONS}

The constitutive equations of a bianisotropic material can be written in the $E-B$ formulation as follows

$$
\begin{aligned}
\mathbf{D}(\mathbf{r}, \omega) & =\overline{\bar{\varepsilon}}(\mathbf{r}, \omega) \mathbf{E}(\mathbf{r}, \omega)+\overline{\overline{\boldsymbol{\xi}}}(\mathbf{r}, \omega) \mathbf{B}(\mathbf{r}, \omega), \\
\mathbf{H}(\mathbf{r}, \omega) & =\overline{\overline{\boldsymbol{\zeta}}}(\mathbf{r}, \omega) \mathbf{E}(\mathbf{r}, \omega)+\overline{\overline{\boldsymbol{\nu}}}(\mathbf{r}, \omega) \mathbf{B}(\mathbf{r}, \omega) .
\end{aligned}
$$

The electric displacement $\mathbf{D}(\mathbf{r}, \omega)$ and the magnetic field $\mathbf{H}(\mathbf{r}, \omega)$ are obtained from the electric field $\mathbf{E}(\mathbf{r}, \omega)$ and the magnetic induction $\mathbf{B}(\mathbf{r}, \omega)$, by means of the tensors $\overline{\bar{\varepsilon}}, \overline{\bar{\xi}}$, $\overline{\bar{\zeta}}$ and $\overline{\bar{\nu}}$. The tensors depend on both the position vector $\mathbf{r}$ and on the angular frequency $\omega$, as is the case for inhomogeneous and/or dispersive materials. If in particular both $\overline{\bar{\xi}}(\mathbf{r}, \omega)$ and $\overline{\overline{\boldsymbol{\zeta}}}(\mathbf{r}, \omega)$ are zero, the bianisotropic material modeled by Eqs. (1) and (2) is a standard anisotropic material, characterized only by the two tensors $\overline{\bar{\varepsilon}}(\mathbf{r}, \omega)$ and $\overline{\overline{\boldsymbol{\nu}}}(\mathbf{r}, \omega)$.

The energetic approach introduced in [4] for discretizing constitutive equations in FIT of anisotropic materials can be extended to bianisotropic materials as follows. Let the spatial region $\Omega$ be discretized by a polyhedral primal grid $\mathcal{G}$ and its barycentric dual grid $\tilde{\mathcal{G}}$. Let $e(\omega)$ be the array of the circulations $e_{i}(\omega)$, with $i=1, \ldots n_{e}$, of the electric field $\mathbf{E}(\mathbf{r}, \omega)$ along the $n_{e}$ edges of the primal grid $\mathcal{G}$. Let $\boldsymbol{b}(\omega)$ be the array of the fluxes $b_{i}(\omega)$, with $i=1, \ldots, n_{f}$, of the magnetic induction $\mathbf{B}(\mathbf{r}, \omega)$ across the faces of the primal grid $\mathcal{G}$. In a similar let $\tilde{\boldsymbol{d}}(\omega)$ be the array of the fluxes $\tilde{d}_{i}(\omega)$, with $i=1, \ldots n_{\tilde{f}}$, of the electric displacement $\mathbf{D}(\mathbf{r}, \omega)$ across the $n_{\tilde{f}}=n_{e}$ faces of the dual grid $\tilde{\mathcal{G}}$. Finally let $\tilde{\boldsymbol{h}}(\omega)$ be the array of the circulations $\tilde{h_{i}}(\omega)$, with $i=1, \ldots, n_{\tilde{e}}$, of the magnetic field $\mathbf{H}(\mathbf{r}, \omega)$ along the $n_{\tilde{e}}=n_{f}$ edges of the dual grid $\tilde{\mathcal{G}}$.

Let $\mathbf{w}_{e}^{i}(\mathbf{r})$, with $i=1, \ldots n_{e}$, be the same piecewise uniform vector functions introduced in [4] for tetrahedral grids and extended to polyhedral grids in [5], [6] for approximating the electric field $\mathbf{E}(\mathbf{r}, \omega)$ from the circulations $e_{i}(\omega)$. Similarly let $\mathbf{w}_{f}^{i}(\mathbf{r})$, with $i=1, \ldots f$, be the same piece-wise uniform vector functions introduced in [4] for approximating the magnetic induction $\mathbf{B}(\mathbf{r}, \omega)$ from the fluxes $b_{i}(\omega)$. Using all these basis functions the following quantities can be computed

$$
\begin{aligned}
& m_{\varepsilon}^{i j}(\omega)=\int_{\Omega} \mathbf{w}_{e}^{i}(\mathbf{r}) \cdot \boldsymbol{\varepsilon}(\mathbf{r}, \omega) \mathbf{w}_{e}^{j}(\mathbf{r}) d \mathbf{r}, \\
& m_{\boldsymbol{\xi}}^{i j}(\omega)=\int_{\Omega} \mathbf{w}_{e}^{i}(\mathbf{r}) \cdot \boldsymbol{\xi}(\mathbf{r}, \omega) \mathbf{w}_{f}^{j}(\mathbf{r}) d \mathbf{r}, \\
& m_{\boldsymbol{\zeta}}^{i j}(\omega)=\int_{\Omega} \mathbf{w}_{f}^{i}(\mathbf{r}) \cdot \boldsymbol{\zeta}(\mathbf{r}, \omega) \mathbf{w}_{e}^{j}(\mathbf{r}) d \mathbf{r}, \\
& m_{\boldsymbol{\nu}}^{i j}(\omega)=\int_{\Omega} \mathbf{w}_{f}^{i}(\mathbf{r}) \cdot \boldsymbol{\nu}(\mathbf{r}, \omega) \mathbf{w}_{f}^{j}(\mathbf{r}) d \mathbf{r},
\end{aligned}
$$

which are the elements respectively of the $n_{e} \times n_{e}$ matrix $\mathbf{M}_{\varepsilon}(\omega)$, of the $n_{e} \times n_{f}$ matrix $\mathbf{M}_{\boldsymbol{\xi}}(\omega)$, of the $n_{f} \times n_{e}$ matrix $\mathbf{M}_{\zeta}(\omega)$ and of the $n_{f} \times n_{f}$ matrix $\mathbf{M}_{\nu}(\omega)$. Matrices $\mathbf{M}_{\varepsilon}(\omega)$ and $\mathbf{M}_{\nu}(\omega)$ have already been introduced for FIT in [4], while matrices $\mathbf{M}_{\boldsymbol{\xi}}(\omega)$ and $\mathbf{M}_{\boldsymbol{\zeta}}(\omega)$ are here introduced for the first time.

Thanks to the geometric properties of the basis functions $\mathbf{w}_{e}^{i}(\mathbf{r})$ and $\mathbf{w}_{f}^{i}(\mathbf{r})$ [4] the following result can be proven.

Property 1: The equations

$$
\begin{aligned}
& \tilde{\boldsymbol{d}}(\omega)=\mathbf{M}_{\boldsymbol{\varepsilon}}(\omega) \boldsymbol{e}(\omega)+\mathbf{M}_{\boldsymbol{\xi}}(\omega) \boldsymbol{b}(\omega) \\
& \tilde{\boldsymbol{h}}(\omega)=\mathbf{M}_{\boldsymbol{\zeta}}(\omega) \boldsymbol{e}(\omega)+\mathbf{M}_{\boldsymbol{\nu}}(\omega) \boldsymbol{b}(\omega)
\end{aligned}
$$

are FIT discretizations of the bianisotropic constitutive equations (1), (2). In fact they exactly transform the circulations of $\mathbf{E}(\mathbf{r}, \omega)$ along the edges of $\mathcal{G}$ and the fluxes of $\mathbf{B}(\mathbf{r}, \omega)$ across 
the faces of $\mathcal{G}$ into the fluxes of $\mathbf{D}(\mathbf{r}, \omega)$ across the faces of $\tilde{\mathcal{G}}$ and the circulations of $\mathbf{H}(\mathbf{r}, \omega)$ along the edges of $\tilde{\mathcal{G}}$, in the hypothesis that $\mathbf{E}(\mathbf{r}, \omega), \mathbf{B}(\mathbf{r}, \omega)$ and $\overline{\bar{\varepsilon}}(\mathbf{r}, \omega), \overline{\boldsymbol{\xi}}(\mathbf{r}, \omega), \overline{\overline{\boldsymbol{\zeta}}}(\mathbf{r}, \omega)$, $\overline{\bar{\nu}}(\mathbf{r}, \omega)$ are uniform in each volume of $\mathcal{G}$.

The discretized constitutive equations of bianisotropic materials are thus consistent extensions of discretized constitutive equations of anisotropic materials within FIT. As it can be proven, these equations preserve the thermodynamic properties [5] of the bianisotropic constitutive equations (1), (2), such as reciprocity and passivity or in particular losslessness. These properties are not only important on a physical ground, but also crucial in numerical analysis since, similarly to the case of anisotropic materials, they ensure a stable discretization.

\section{BIANISOTROPIC FIT FORMULATION}

The discrete constitutive equations of bianisotropic materials are approximate equations that have to be completed by the balance equations characteristic of FIT. Hence, equations in the angular frequency domain $\omega$ are considered, Faraday's equation is discretized in terms of the exact matrix equation

$$
\mathbf{C e}(\omega)=-i \omega \boldsymbol{b}(\omega)
$$

in which $\mathbf{C}$ is the $n_{f} \times n_{e}$ face-edge incidence matrix of the primal grid $\mathcal{G}$. Similarly Ampère-Maxwell's equation is discretized in terms of the exact matrix equation

$$
\tilde{\mathbf{C}} \tilde{\boldsymbol{h}}(\omega)=i \omega \tilde{\boldsymbol{d}}(\omega),
$$

in which $\tilde{\mathbf{C}}=\mathbf{C}^{T}$ is the $n_{\tilde{f}} \times n_{\tilde{e}}$ face-edge incidence matrix of dual grid $\tilde{\mathcal{G}}$. As with anisotropic materials, also with bianisotropic materials, the number of discrete equations (3)-(6) can be reduced by removing discrete variables. In particular, substituting Eqs. (3), (4) into Eq. (6) for $\tilde{\boldsymbol{d}}(\omega)$ and $\tilde{\boldsymbol{h}}(\omega)$, and then substituting Eq. (5) in the obtained equation for $\boldsymbol{b}(\omega)$ it can be written

$$
\begin{aligned}
\left(\mathbf{C}^{T} \mathbf{M}_{\boldsymbol{\nu}}(\omega) \mathbf{C}-i \omega\left(\mathbf{C}^{T} \mathbf{M}_{\boldsymbol{\zeta}}(\omega)+\right.\right. & \\
+ & \left.\left.\mathbf{M}_{\boldsymbol{\xi}}(\omega) \mathbf{C}\right)-\omega^{2} \mathbf{M}_{\boldsymbol{\varepsilon}}(\omega)\right) \boldsymbol{e}(\omega)=\mathbf{0}
\end{aligned}
$$

Boundary conditions have to be treated as usual in FIT. Eq. (7) is an extension of the equations written in the sole discrete variables $e$ for anisotropic materials. It is suitable for applying the preconditioning and iterative methods already used in the case of anisotropic materials.

\section{NUMERICAL EXAMPLE}

The numerical example refers to the waveguide $\Omega$ shown in Fig. 2. Without loss of generality, half of the waveguide is filled with a lossless bi-isotropic material, which is both important from the point of view of practical applications and can be realized with the help of metamaterials. Precisely, all tensors are diagonal, $\overline{\bar{\varepsilon}}$ and $\overline{\bar{\nu}}$ have the properties of vacuum in all $\Omega$, while $\bar{\xi}=\overline{\bar{\zeta}}$ are equal to the purely imaginary scalar $i \kappa$ which is different from zero only within the bi-isotropic material. The waveguide is meshed with tetrahedra, with the basis functions of [4]. The susceptance $\operatorname{Im}(Y)$ at the $T E_{10}$ port of the structure has been evaluated for three grids with 24133 ,

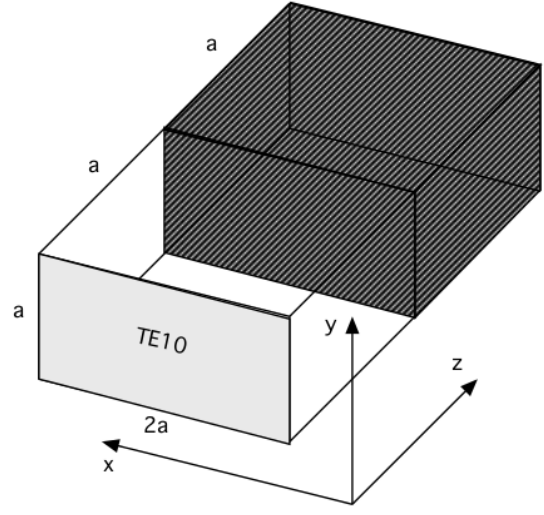

Figure 1: Benchmark problem

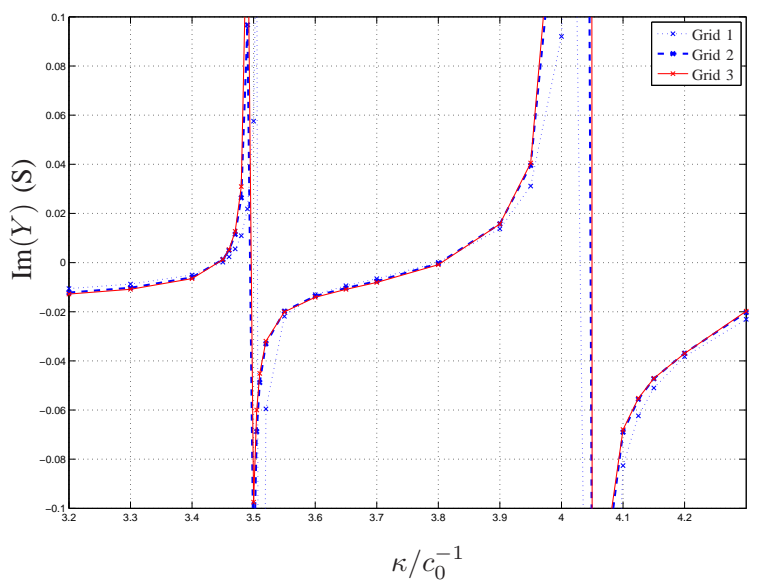

Figure 2: Susceptance $\operatorname{Im}(Y)$ at the $T E_{10}$ port as a function of material parameter $\kappa$, for increasingly refined meshes.

35934 and 55456 edges, respectively. Accurate estimations of $\operatorname{Im}(Y)$ are obtained for the rather coarse grid 2, as shown in Fig. 2.

\section{CONCLusions}

The full paper will include proofs of the most important properties of the proposed scheme, details on the implementation of the formulation and imposition of boundary conditions.

\section{REFERENCES}

[1] R. Marqus, F. Medina and R. Rafii-El-Idrissi, "Role of bianisotropy in negative, permeability and left-handed metamaterials," Physical Review B - Condensed Matter and Materials Physics, vol. 65, no. 14, pp. 14444011444406, 2002

[2] J. A. Kongi, "Theorems of bianisotropic media," Proc. of the IEEE, vol. 60, no. 9, pp. 1036-1046, 1972.

[3] P. Fernandes and M. Raffetto, "Well-posedness and finite element approximability of time-harmonic electromagnetic boundary value problems involving bianisotropic materials and metamaterials," Mathematical Models and Methods in Applied Sciences, vol. 19, no. 12, pp. 2299-2335, 2009.

[4] L. Codecasa and M. Politi, "Use of barycentric dual grids for the solution of frequency domain problems by FIT," IEEE Transactions on Magnetics, Vol. 40, No. 2 pp. 1414-1419, 2004.

[5] L. Codecasa and F. Trevisan, "Constitutive equations for discrete electromagnetic problems over polyhedral grids," Journal of Computational Physics, Vol. 225 No. 2, pp. 1894-1918, 2007.

[6] L. Codecasa, R. Specogna, F. Trevisan, "Base functions and discrete constitutive relations for staggered polyhedral grids," Computer Methods in Applied Mechanics and Engineering, Vol. 198, pp. 1117-1123, 2009. 